\title{
Pulmonary inflammatory cells in ventilated preterm infants: effect of surfactant treatment
}

\author{
Shmuel Arnon, Jonathan Grigg, Michael Silverman
}

\begin{abstract}
The aim of this study was to determine the effect of surfactant treatment on the number and distribution of inflammatory cells in bronchoalveolar lavage fluid (BALF) from mechanically ventilated preterm infants over the first week of life in relation to the subsequent development of chronic lung disease (CLD). The study included 25 babies who received surfactant on clinical grounds and 29 babies of similar severity who did not. BALF was collected on days $1,3,5$, and 7 after birth. Cell counts were performed and differentials were calculated on 300 cells. CLD was equally common in both treatment groups. Of the 54 infants, 29 (53\%) who developed CLD had a higher incidence of patent ductus arteriosus and air leak and needed a higher concentration of inspired oxygen on the fifth and seventh days of life. Babies who developed CLD had more polymorphonuclear leucocytes and fewer macrophages on days 5 and 7 than those who recovered. Surfactant treatment was associated with a higher total white cell count on day 3. Between days 3 and 7 , macrophage numbers were higher in surfactant treated babies, whatever the pulmonary outcome. This data suggests that CLD was associated with persistence of high numbers of polymorphonuclear leucocytes in BALF at the end of the first week. Surfactant treatment caused a persistent increase in macrophage numbers. The association between persistent neutrophilia and CLD was unaffected by surfactant treatment.
\end{abstract}

(Arch Dis Child 1993; 69: 44-48)

Since the introduction of the term bronchopulmonary dysplasia by Northway, ${ }^{1}$ attempts have been made to identify features which might distinguish those preterm infants who subsequently develop chronic lung disease (CLD) of prematurity from those who recover from the initial phase of respiratory distress syndrome. Several groups have attempted to do this by examining the numbers and distribution of inflammatory cells obtained by bronchoalveolar lavage during the first weeks of life. ${ }^{2} 3$

Under experimental conditions, inflammatory cells obtained by bronchoalveolar lavage have been used as a marker of lung damage and repair. ${ }^{4}$ In human infants with respiratory distress syndrome Ogden et al showed that there were very few cells in bronchoalveolar lavage fluid (BALF) on the first day of life, whereas at 48 and 96 hours cell counts, mainly polymorphonuclear leucocytes, increased in all infants. Numbers of polymorphonuclear leucocytes remained high only in those who later developed bronchopulmonary dysplasia by Northway's definition. ${ }^{3}$ The factors which link excess of polymorphonuclear leucocytes with subsequent lung disease are speculative. It is clear that inflammatory cell activation takes place in the newborn lung as numerous mediators and cytokines have been identified in BALF and blood. ${ }^{56}$ At the same time, preterm babies exhibit reduced capacity for host defence that may enhance the damaging effects of such mediators. ${ }^{237}$

Macrophages can also be identified in BALF in the first week of life. Their appearance in the lungs of newborn monkeys has been found to correlate with the occurrence of surface active material. This association may explain the observation that the number of macrophages was greater at birth in term monkeys than in preterm and that the number increased with postnatal age in both term and preterm monkeys without hyaline membrane disease but not in preterms with hyaline membrane disease. ${ }^{8}$ Macrophages have been shown to engulf components of surfactant, natural or artificial. In the process, the phagocytic and chemotactic capacity of the macrophages may be impaired. ${ }^{9}$

The aim of this study was to determine the effect of surfactant treatment on the population of inflammatory cells obtained by bronchoalveolar lavage over the first few postnatal days in intubated preterm infants with respiratory distress syndrome and to relate changes in cell counts to the development of CLD.

\section{Patients and methods}

Fifty four infants were included in an open study. All were born at less than 34 weeks of gestation (mean 29.1 weeks) and developed respiratory distress syndrome requiring mechanical ventilation after birth. Of these, 25 received natural porcine surfactant (Curosurf, Chiesi Farmaceutici) and 29 did not. The preparation was given as rescue treatment within 72 hours of birth, in a dose of 100 or $200 \mathrm{mg} / \mathrm{kg}$ of phospholipid with a volume of 1.25 or $2.5 \mathrm{ml} / \mathrm{kg}$, followed by up to four more doses over 48 hours. The patients who received surfactant were participants in an open clinical trial of two therapeutic regimens of surfactant administration. Those who did not receive surfactant treatment were studied in the few months immediately before the introduction of surfactant in our neonatal intensive care unit. They comprised all of the infants who fulfilled the study criteria, on 
Patient data by group, figures are mean (SD) or number (\%)

\begin{tabular}{|c|c|c|c|c|}
\hline & \multicolumn{2}{|l|}{ No surfactant } & \multicolumn{2}{|l|}{ Surfactant } \\
\hline & No $C L D$ & $C L D$ & No $C L D$ & $C L D$ \\
\hline $\begin{array}{l}\text { No of infants } \\
\text { Birth weight }(\mathrm{g}) \\
\text { Dexamethasone (prenatal) } \\
\text { Apgar score }(5 \mathrm{~min}) \\
\text { Mean PIP day } 1\left(\mathrm{~cm} \mathrm{H}_{2} 0\right) \\
\text { Mean } \mathrm{FIO}_{2} \text { day } 1(\%) \\
\text { Mean } \mathrm{PIP} \text { day } 5\left(\mathrm{~cm} \mathrm{H}_{2} 0\right) \\
\text { Mean FiO day } 5(\%) \\
\text { Patent ductus arteriosus } \\
\text { Air leak } \\
\text { Dexamethasone (beyond two weeks) } \\
\text { Sepsis (beyond } 10 \text { days) } \oint\end{array}$ & $\begin{array}{c}14(26) \\
1012(239) \\
6(42) \\
7 \cdot 1(1 \cdot 6) \\
20 \cdot 2(2 \cdot 1) \\
45 \cdot 6(10 \cdot 8) \\
18 \cdot 4(4 \cdot 3) \\
41(8 \cdot 3) \\
4(28) \\
3(21) \\
10(71) \\
9(64)\end{array}$ & $\begin{array}{c}15(28) \\
957(82) \\
8(53) \\
7 \cdot 2(1 \cdot 1) \\
21 \cdot 8(3 \cdot 6) \\
50 \cdot 8(16 \cdot 4) \\
24 \cdot 3(6 \cdot 2) \\
70(15 \cdot 6) \dagger \\
5(33) \\
6(40) \dagger \\
15(100) \\
11(73) \dagger\end{array}$ & $\begin{array}{c}11(20) \\
1105(195) \\
5(45) \\
8 \cdot 1(0 \cdot 7) \\
25 \cdot 5(3 \cdot 4)^{\star} \\
42 \cdot 1(14 \cdot 5) \\
21 \cdot 8(4 \cdot 5) \\
35(6 \cdot 4) \\
5(45) \\
1(9) \\
9(81) \\
5(45)\end{array}$ & $\begin{array}{c}14(26) \\
1117(285) \\
7(50) \\
7 \cdot 6(1 \cdot 2) \\
24 \cdot 8(2 \cdot 5)^{\star} \\
54 \cdot 4(20 \cdot 2) \\
5 \cdot 8(3 \cdot 7) \\
58(6 \cdot 4) \dagger \\
11(78) \ddagger \\
4(28) \dagger \\
14(100) \\
13(92) \dagger\end{array}$ \\
\hline
\end{tabular}

$\mathrm{PIP}=$ peak inspiratory pressure; $\mathrm{FIO}_{2}=$ fractional inspired oxygen concentration.

${ }^{\star} p<0.05$ compared with no surfactant group; $\nmid p<0.05$ compared with no CLD group; $\neq p<0.05$ compared with no surfactant/no CLD group; Spositive bacterial culture of blood, urine, or cerebrospinal fluid.
Differential counts of macrophages and polymorphonuclear leucocytes were calculated from 300 cells, on centrifuge preparations (Cytospin 2, Shandon Products Ltd) stained with Diff Quick (Merz and Dade AG). A sample of BALF was analysed for aerobic and anaerobic bacteria by standard microbiological techniques.

\section{STATISTICAL ANALYSIS}

Before analysis, BALF cell counts were log transformed from a skewed to a normal distribution. Discrete variables were compared by unpaired $t$ test with Bonferoni correction for multiple comparisons. We did not attempt to divide the surfactant patients into small subsets according to therapeutic group (high or low dose) because of the small numbers involved.

whom BALF samples had been collected over the first week of life. There was no other form of selection and the results of BALF analysis were only known after the groups had been defined. The whole study took place over a 12 month period during which other management policies did not change.

The diagnosis of respiratory distress syndrome was based on the findings of respiratory distress with an indicative chest radiograph. CLD was defined as a need for supplemental oxygen for at least 28 days, still present at 36 weeks' postconceptional age. ${ }^{10}$ The following infants were excluded from the study: those who had a history of prolonged rupture of membranes, amnionitis, maternal fever and antibiotic treatment, positive blood or BALF cultures, a diagnosis of pneumonia or sepsis before 10 days of age, or who died before 28 days of age. Babies were followed up until death or discharge from the neonatal unit.

Bronchoalveolar lavage was performed on all babies at $1,3,5$, and 7 days of age. The first sample was collected after surfactant administration.

\section{BRONCHOALVEOLAR LAVAGE}

The infant's head was turned to the left in order to ensure a consistent sampling site in the right lower zone. ${ }^{11}$ An aliquot of $1 \mathrm{ml} / \mathrm{kg}$ normal saline was introduced down the right main bronchus via an end hole, 5 French suction catheter which had been wedged lightly. Bronchoalveolar lavage fluid was immediately aspirated and collected into a sterile container. The procedure was then repeated once and the two aspirates were pooled. Parental consent was obtained for this modified nursing procedure, which was conducted at the time of routine endotracheal suctioning by nursing staff. The total duration of the procedure was less than one minute. Samples were excluded from further analysis if there was visible bloodstaining or if the volume recovered was $<30 \%$ of the volume instilled.

The processing of BALF has been described previously. ${ }^{12}$ Briefly, pooled BALF was filtered through a muslin gauze to remove mucus and the total number of cells determined by haemocytometer (BALF cells $\times 10^{4}$ ). Cell viability was assessed by trypan blue exclusion.

\section{Results}

Of the 54 infants, 29 (53\%) developed CLD according to the above criteria, $15 / 29$ control infants and 14/25 who had received surfactant. Babies who developed CLD had a higher incidence of patent ductus arteriosus and air inspired oxygen on the fifth (table) and seventh days of life $(p<0.05)$. There were few significant differences between the surfactant and non-surfactant treated infants except for the higher first day mean peak inspiratory pressure and subsequently a higher incidence of clinically diagnosed patent ductus arteriosus requiring diuretic or indomethacin treatment in the surfactant group (table). There was no significant difference in mean inspired oxygen concentration, ventilatory rate, or level of positive end expiratory pressure in the first week of life, between the two groups.

Beyond the one week sampling period, most infants received a course of dexamethasone; those who subsequently developed CLD had a greater incidence of bacterial sepsis (positive blood, urine, or cerebrospinal fluid culture) beyond the sampling period of one week (table).

\section{BRONCHOALVEOLAR LAVAGE}

The volume of fluid recovered did not vary between groups or between study days. The overall mean (SD) proportion recovered from over 200 bronchoalveolar lavage procedures was $55.5(7 \cdot 6) \%$, with a mean volume of sterile. The numbers of samples rejected because of bloodstaining or small volume was 19 out of a total of 216 samples.

There was no difference in the total number of inflammatory cells between babies who developed CLD and those who did not. However, babies who developed CLD had more polymorphonuclear leucocytes and fewer macrophages on days 5 and 7 than those who recovered without CLD (fig 1). Surfactant treatment had a significant effect on the BALF cell population on days 3-5 (fig 2). Total cell counts as well as polymorphonuclear leak, and needed a higher concentration of $1 \cdot 1 \mathrm{ml} / \mathrm{kg}$. All samples were bacteriologically 
leucocytes and macrophage cell numbers were greater in the treatment group on day 3 , the higher macrophage count persisting until day 5.
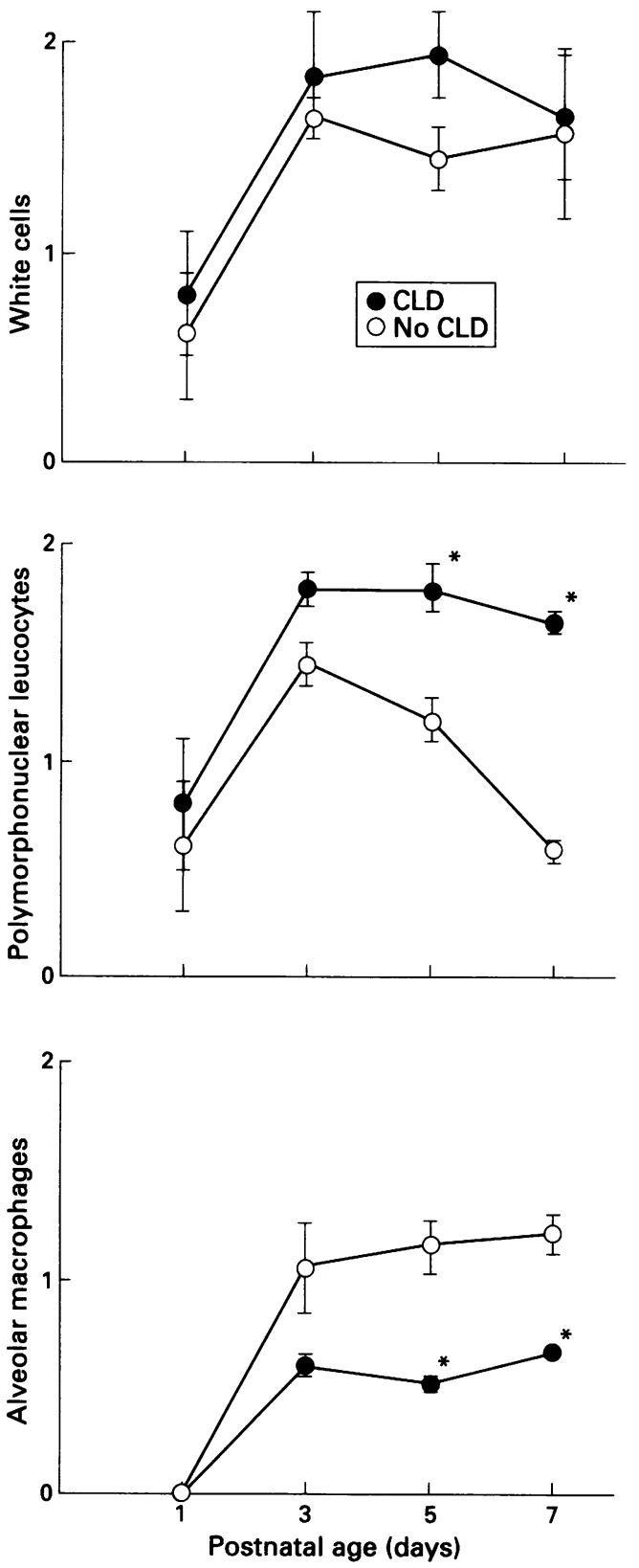

Figure 1 BALF inflammatory cells (mean (SD) $\log _{10} \times 10^{4} / B A L F$ sample) in the first week of life; ${ }^{\star} p<0.01$, compared with no CLD group.

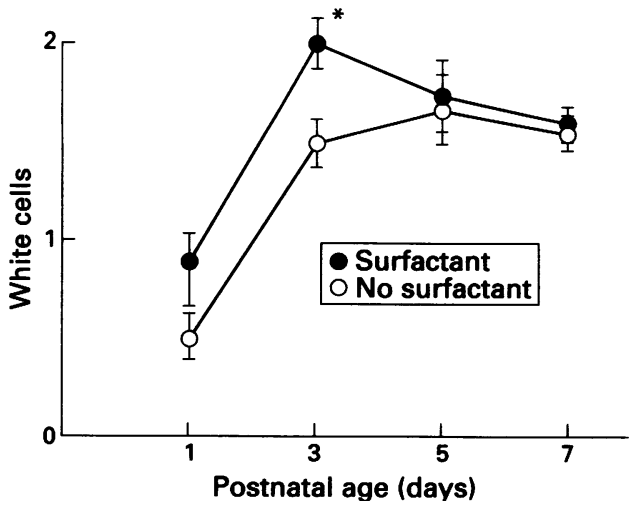

Figure 2 White cell count (mean (SD) $\log _{10} \times 10^{4} / B A L F$ sample) in surfactant treated babies and those who received no surfactant in the first week of life; ${ }^{\star} p<0.01$, compared with no surfactant group.
When the data for the four groups (divided according to surfactant or no surfactant and CLD or no CLD) were examined separately (fig 3), only a persistently high count of polymorphonuclear leucocytes at day 7 was definitely associated with the subsequent development of CLD. Macrophage cell numbers were higher overall in surfactant treated babies compared with untreated babies on days 3-7 and were persistently low at these times only in the infants who did not receive surfactant but subsequently developed CLD (fig 3).

\section{Discussion}

This study has confirmed the association between persistence of polymorphonuclear leucocytes in BALF and the subsequent development of CLD in mechanically ventilated preterm infants with simple respiratory distress syndrome. ${ }^{23}$ The association was unaffected by a significant but transient effect of natural surfactant rescue treatment on inflammatory cell numbers in BALF. The major effect of surfactant treatment appeared to be to accelerate the appearance of macrophages in BALF, independently of later pulmonary outcome.

Although these results are consistent with the hypothesis that the persistence of a high ratio of polymorphonuclear leucocytes: macrophages is associated with subsequent CLD, 23 our data would suggest that the use of a ratio masks the importance of polymorphonuclear leucocytes and conversely, lends undue importance to macrophage counts which we found to be of low predictive value.

\section{METHODOLOGY}

Some important issues related to the methodology of bronchoalveolar lavage in neonates must be considered in order to interpret our results. As is customary, we have reported total cell counts, unrelated to the precise quantity of epithelial lining fluid which we sampled. Although for solutes such an approach could be inappropriate, ${ }^{12}$ in the present study we were more concerned to assess the total inflammatory cell load accessible by our technique of bronchoalveolar lavage. The proportion of BALF recovered was similar for the four groups on each study day.

We did not attempt to assess the distribution of cell types in the lung, a potentially important factor in relation to the site of lung damage and hence outcome. In previous work we showed that a second aliquot of BALF results in a higher proportion of macrophages than the first one. ${ }^{12}$ This may be explained by the fact that the trachea and large airways are predominantly occupied by polymorphonuclear leucocytes during mechanical ventilation and that macrophages predominate in the lung periphery. Changes in the number of macrophages obtained by bronchoalveolar lavage may therefore reflect the changes in the small airways and alveoli. Changes in counts of polymorphonuclear leucocytes may result from 

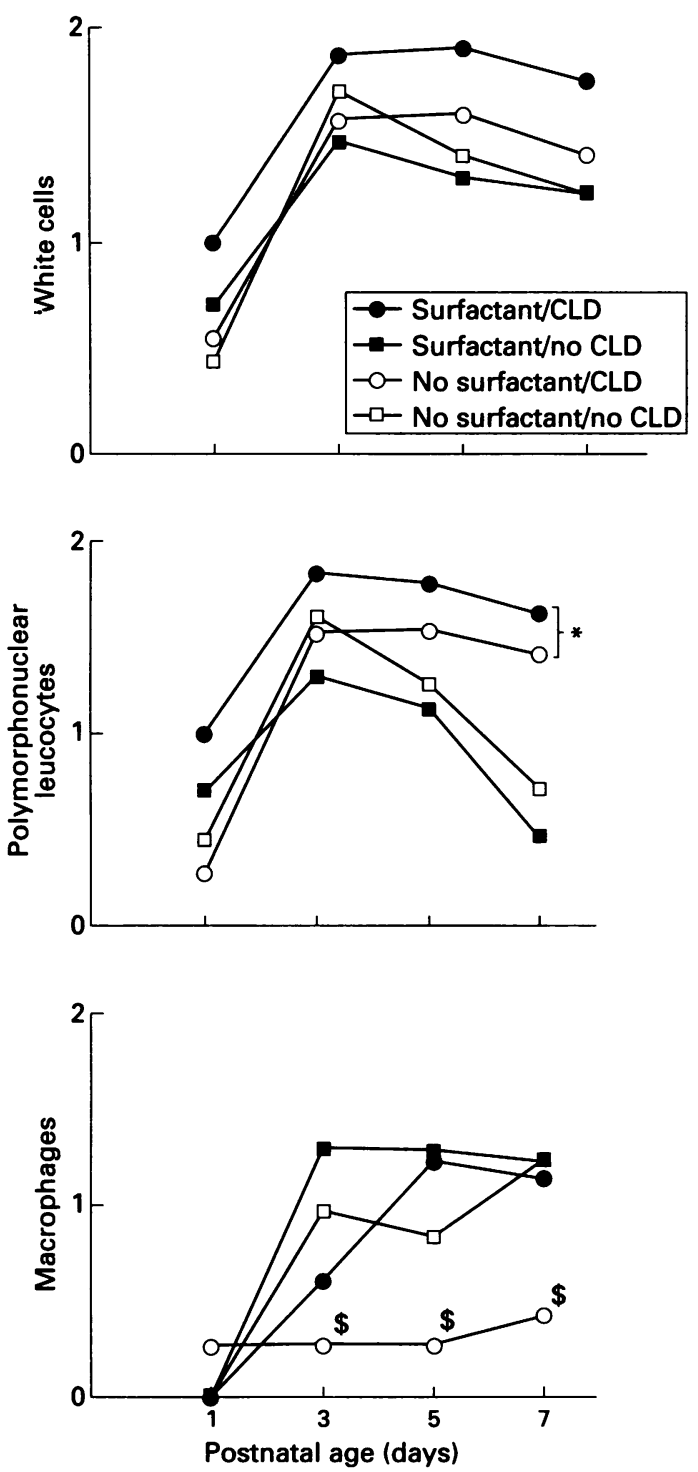

Figure $3 B A L F$ inflammatory cells (mean $\log _{10} \times 10^{4}$ ) $B A L F$ sample) in the first week of life, in relation to $C L D$ and surfactant treatment. ${ }^{\star} D i f f e r e n c e$ between infants with and without $C L D ; p<0.01$. \$Difference between $B A L F$ macrophage counts of infants with CLD (without surfactant) and all other groups was significant on days 3 , 5 , and $7 ; p<0 \cdot 01$.

inflammation of the segmental and subsegmental airways and this may explain their association with persistent CLD, largely a disorder of airways.

Simple suction bronchoalveolar lavage is not directed to any particular lung site. Nor is it possible to prove that the two samples from each infant originated from the same segment, although this seems likely. ${ }^{11} 12$ Schmekel and coworkers, assessing 12 healthy subjects for the interlobar variation of neutrophils, eosinophils and solutes, observed interlobar consistency in the numbers and proportions of neutrophils and eosinophils but not for the solutes. ${ }^{13}$ Other studies have also shown good interlobar consistency in patients with non-focal disease on the chest radiograph. ${ }^{14} 15$ Respiratory distress syndrome and CLD are by definition generalised lung disorders, so that possible variation in sample site is probably of no significance.

We did not include babies with pre-existing lung conditions such as overt or suspected infection, which could have influenced the inflammatory cell population in the lung. To do so would have rendered the surfactant and control groups non-comparable, as surfactant would not routinely be given to babies with lung disease of infective origin. Although the groups were sequential rather than being based on random assessment of subjects, they were closely comparable before surfactant administration. Over the limited 12 month period of the study, management protocols other than those involving surfactant therapy, had not altered. Thus, although the study did not have randomised design, we feel that the results are valid.

\section{BALF INFLAMMATORY CELLS}

Jacob and coworkers previously reported in newborn monkeys that macrophage numbers were low in utero and increased markedly in the postnatal period in term monkeys without hyaline membrane disease. ${ }^{8}$ They did not increase postnatally in monkeys with hyaline membrane disease that had persistent deficiency in phospholipid surface active material in BALF. Either a common determinant could cause both the increase in alveolar surface active material and in macrophage number, or the increase in one may lead to the increase in the other. ${ }^{16} 17$

Our study strongly suggests that very soon after administration of an exogenous natural surfactant preparation, macrophage numbers increase in BALF. The rapidity of the change implies a specific chemoattractant effect. In our study the surfactant used was a natural extract, prepared from minced porcine lung. It contained $99 \%$ polar lipids, predominantly phospholipid, and only $1 \%$ lipophilic low molecular weight proteins. ${ }^{18}$ The postnatal rise in macrophages seems likely therefore to be a response to phospholipid in the epithelial lining fluid of the neonatal lung, whether endogenous or exogenous in origin.

An alternative explanation, kindly suggested by a reviewer, is that after surfactant, a larger alveolar component, and therefore more macrophages, may have been released. This is possible, although we might have expected a drop in the BALF volume under these circumstances. However, there was no difference in the proportion returned. Other possible explanations for enhanced cellularity are that cellular adherence to the epithelium was reduced by surfactant treatment, selectively releasing alveolar macrophages, or that small amounts of lipid soluble cytokines in natural surfactant extracts lead to increased cellular influx. There is no evidence to support any of these hypotheses.

Ogden $e t a l^{3}$ and Merritt et $a l^{2}$ analysed cells in tracheal aspirates and BALF collected from human newborns. BALF macrophage counts were significantly increased in respiratory distress syndrome at 4 days, compared with control subjects, but not in those who subsequently developed bronchopulmonary dysplasia. In their study, the control group consisted of term and preterm infants without 
respiratory illness, which may indicate that the dynamic process of recruiting macrophages not only depends on the presence of surfactant but also on processes of damage and repair.

Macrophages and polymorphonuclear leucocytes participate both in lung defence and injury. ${ }^{19}$ The macrophage is a versatile cell with paradoxical effects, able to release oxidants, proteolytic enzymes, mediators, ${ }^{20}$ and to ingest surfactant particles ${ }^{9}$ but also able to secrete antioxidants, antiproteases and inhibitors of certain cytokines. ${ }^{19}$ It is also involved in the resolution of injury by ingestion of apoptotic neutrophils. ${ }^{21} 22$ The pathogenesis of CLD is complex and many factors are involved in the evolution of this disease. Our data did not show a simple relationship between macrophage numbers over the first week of life and subsequent pulmonary outcome.

\section{CONCLUSIONS}

We have confirmed previous observations showing that recovery from the initial phase of respiratory distress syndrome is associated with marked decline in the proportion and number of polymorphonuclear leucocytes at 5 and 7 days of age. CLD was associated with a persistence of neutrophils in BALF at the end of the first week of life. The administration of natural surfactant extract produced a transient rise in total white cell numbers and a persistent increase in macrophages, so that neither macrophage counts over the first week nor the polymorphonuclear leucocyte to macrophage ratio were predictive of CLD. The nature of the macrophage response to exogenous surfactant, and the role of the enhanced numbers of macrophages in the balance of inflammatory lung damage and resolution, remain to be elucidated.

Dr Grigg was supported by a grant from Action Research and Dr Arnon in part by Astra Pharmaceuticals Ltd.

We are grateful to Dr John Savill for providing laboratory space and facilities.

1 Northway WH Jr, Rosan RC, Porter DY. Pulmonary disease following respirator therapy of hyaline membrane disease: bronchopulmonary dysplasia. N Engl f Med 1967; 276: $257-68$.
2 Merritt TA, Cochrane CG, Holcomb K, et al. Elastase and alphal-proteinase inhibitor activity in tracheal aspirates during respiratory distress syndrome. Role of inflammation in the pathogenesis of bronchopulmonary dysplasia. f Clin Invest 1983; 72: 656-66.

3 Ogden BE, Murphy SA, Saunders GC, Pathak D, Johnson JD. Neonatal lung neutrophils and elastase/proteinase inhibitor imbalance. Am Rev Respir Dis 1984; 130: 817-21.

4 Davis JM, Dickerson B, Methay L, Penney DP. Differential effects of oxygen and barotrauma on lung injury in the neonatal piglet. Pediatr Pulmonol 1991; 10: 157-63.

5 Grigg JM, Barber A, Silverman M. Increased levels of bronchoalveolar lavage fluid interleukin-6 in preterm ventilated infants after prolonged rupture of membranes Am Rev Respir Dis 1992; 145: 782-6.

6 Miller LC, Isa S, LoPreste G, Schaller JC, Dinarello CA. Neonatal interleukin- $1 \beta$, interleukin- 6 and tumor necrosis factor: cord blood levels and cellular production. f Pediatr 1990; 117: 961-5.

7 Grigg J, Barber A, Silverman M. Bronchoalveolar lavage fluid glutathione in intubated premature infants. Arch Dis Child 1993; 69: 49-51.

8 Jacobs RF, Wilson CB, Palmer S, et al. Factors related to the appearance of alveolar macrophages in the developing lung. Am Rev Respir Dis 1985; 131: 548-53.

9 Sherman MP, D'Ambola JB, Aeberhard EE, Barrett CT. Surfactant therapy of newborn rabbits impairs lung macrophage bactericidal activity. F Appl Physiol 1988; 65: 137-45

10 Shennan AT, Dunn MS, Ohisson A, Lennox K, Hoskins E. Abnormal pulmonary outcomes in premature infants. Prediction from oxygen requirement in the neonatal period. Pediatrics 1988; 82: 527-32.

11 Placzek M, Silverman M. Selective placement of bronchial suction catheters in intubated neonates. Arch Dis Child 1983; 58: 829-30.

12 Grigg J, Arnon S, Silverman M. Functional processing of sequential bronchoalveolar lavage fluid from intubated babies. Eur Respir f 1992; 5: 727-32.

13 Schmekel B, Blom-Bulow B, Hornblad Y, et al. Granulocytes and their secretory products, myeloperoxidase and
eosinophil cationic protein, in bronchoalveolar lavage eosinophil cationic protein, in bronchoalveolar lavage fluid from two lung

14 Peterson MW, Nugent KM, Jolles H, Monick M, Hunninghake GW. Uniformity of bronchoalveolar lavage in patients with pulmonary sarcoidosis. Am Rev Respir Dis 1988; 137: 79-84.

15 Garcia JGN, Wolven RG, Garcia PL, Keogh BA. Assessment of interlobar variation of bronchoalveolar Assessment of interlobar variation of bronchoalveolar Rev Respir Dis 1986; 133: 444-9.

16 Sieger L. Pulmonary alveolar macrophages in pre and postnatal rabbits. Fournal of the Reticuloendothelial Society 1978; 23: 389-95.

17 Bellanti JA, Nerurkar LS, Zeligs BJ. Host defences in the fetus and neonate: studies of the alveolar macrophage during maturation. Pediatrics 1979; 64: 726-39.

18 Yee WFH, Scarpelli EM. Surfactant replacement therapy. Pediatr Pulmonol 1991; 11: 65-80.

19 Sibille Y, Reynolds HY. Macrophages and polymorphonuclear neutrophils in lung defence and injury. $\mathrm{Am}$ Rev Respir Dis 1990; 141: 471-501.

20 Stenmark KR, Eyzaguire M, Westcott JY, Henson PM, Murphy RC. Potential role of eicosanoids and PAF in the pathophysiology of bronchopulmonary dysplasia. Am Rer Respir Dis 1987; 136: 770-2.

21 Wyllie AH, Kerr JFR, Currie AR. Cell death: the significance of apoptosis. Int Rev Cytol 1980; 68: 251-306.

22 Grigg JM, Savill JS, Sarraf C, Haslett C, Silverman M Neutrophil apoptosis and clearance from neonatal lungs. Lancet 1991; 338: 720-2. 